\title{
A Novel Non-contact Measurement Method for the Detection of Current Flowing Through Concealed Conductors
}

\author{
Yang Fan ${ }^{1}$, Liu Kai ${ }^{1}$, Zhu Liwei ${ }^{2}$, Hu Jiayuan ${ }^{2}$, Wang Xiaoyu ${ }^{2}$, \\ Shen Xiaoming ${ }^{2}$, Luo Hanwu', and Ammad Jadoon ${ }^{3}$ \\ ${ }^{1}$ State Key Laboratory of Power Transmission Equipment \& System Security and New Technology, \\ Chongqing University Chongqing, China \\ ${ }^{2}$ State Grid Zhejiang Electric Power Company Metering Center, Hangzhou, China \\ ${ }^{3}$ Maintenance Branch of East Inner Mongolia Electric Power Company Limited, Hohhot 010020, China
}

(Received 31 August 2016, Received in final form 23 December 2016, Accepted 26 December 2016)

\begin{abstract}
In order to detect the current flowing through concealed conductor, this paper proposes a new method based on derivative method. Firstly, this paper analyzes the main peak characteristic of the derivative function of magnetic field generated by a current-carrying conductor, and a relationship between the current flowing through the conductor and the main peak of the derivative function is obtained and applied to calculate the current. Then, the method is applied to detect the conductor current flowing through grounding grids of substations. Finally, the numerical experimental and field experiment verified the feasibility and accuracy of the method, and the computing results show that the method can effectively measure the conductor current of grounding grids with low error, and the error is within $5 \%$.
\end{abstract}

Keywords : current detection, derivative method, grounding grids, magnetic field

\section{Introduction}

With the wide application of electric power, there are lots of current-carrying conductor in practical engineering, which are hidden where it is difficult to measure current, such as buried cable, submarine cable [1], buried pipeline $[2,3]$ or grounding grids of substation $[4,5]$. Generally, in order to evaluate the running status or safety situation of conductor, the current flowing through the conductor need be known. Hence, it is significant to propose a non-contact measurement method to detect the conductor current.

The grounding grids in substation can guarantee safety operation of the substation and has a vital significance for the substation [6]. When there is lightning current or failure current in power grid, grounding grids provide a discharging channel to current [7], hence the conductive ability of each conductor of grounding grid is of great importance as the grounding grid is responsible for the stable operation of the power system, safety of the power

(C)The Korean Magnetics Society. All rights reserved.

*Corresponding author: Tel: +86-18875207852

Fax:+86-023-65111935, e-mail: liukai2015@cqu.edu.cn the power equipment and the personnel working at power grid. Generally grounding grids are made up of iron or galvanized iron and corrosion can occur with the passage of time. Mastering the conductive ability of each conductor of grounding grid can provide references for diagnosing the corrosion state of grounding grid [8]. However, Typically, the grounding grid is made out of bare copper or iron conductors, which are buried about 0.3-2 m deep under the ground, it is difficult to directly measure the current flowing through conductor of grounding grid by normal methods [9]. Ma et al. [10] inject the square wave current with high frequency $(\sim 500 \mathrm{~Hz})$ and high current ( $\sim 10 \mathrm{~A}$ maximum) into the grid and measure the surface magnetic induction intensity to investigate grounding grid integrity. Dawalibi [11] injected a 100-A direct current into the down-lead wire and measured the surface magnetic field to explore the break point diagnosis of grounding grids based on electromagnetic theory. Liu et al. [12] injected a current of $300 \mathrm{~Hz}$ into the down-lead conductors, to measure the surface magnetic induction intensity, and developed a complete detecting system for corrosion or break point diagnosis. Zhang et al. also use the electromagnetic method to diagnosis of breaks in substation's grounding grid [13]. Above method all directly measure 
the magnetic field to explore the topology, corrosion state and break-point of grounding grid. However, the research about current detection of grounding grid is less.

The paper is organized as follows: the mathematical method for detecting conductor current will be introduced and a relationship between the current flowing through conductor and the main peak of derivative function is obtained. Then, a numerical experimental and a field experiment are used to verify the feasibility and accuracy of the method. Finally, the method is applied to detect the current flowing though the conductor of grounding grid and the conclusion ends the paper.

\section{Measurement Method for Current Detection}

Grounding grids of substation are composed by a lot of conductors in finite length. As shown in Fig. 1, a single current-carrying conductor $M N$ is buried in the xoy plane under the ground, the length of the conductor is $L$, and the xoy plane is $h$ meters away from the ground. The conductor is on the $x$-axis. The length of $O M$ is $L_{1}$, the length of $O N$ is $L_{2}$, the current flowing through the conductor is $I$. There is a point $P$, the vertical distance from $P$ to the conductor is $\rho$, the angles between $O P$ and $x$-axis, $N P$ and $M P$ are $\theta, \varphi_{1}$ and $\varphi_{2}$ respectively. The soil of ground is assumed as homogeneous medium with single layer and the permeability is $\mu$. The magnetic flux density at point $P$ is $B$, and the $x$ component and $z$ component are $B_{x}, B_{z}$ respectively. Due to the high resistivity of soil, the conductor's leakage current in the soil can be ignored.

According to the Biot-savart law, the $B_{z}(x)$ can be described,

$$
B_{z}(x)=\frac{\mu I}{4 \pi} \frac{x}{h^{2}+x^{2}}\left(\sin \varphi_{1}+\sin \varphi_{2}\right)
$$

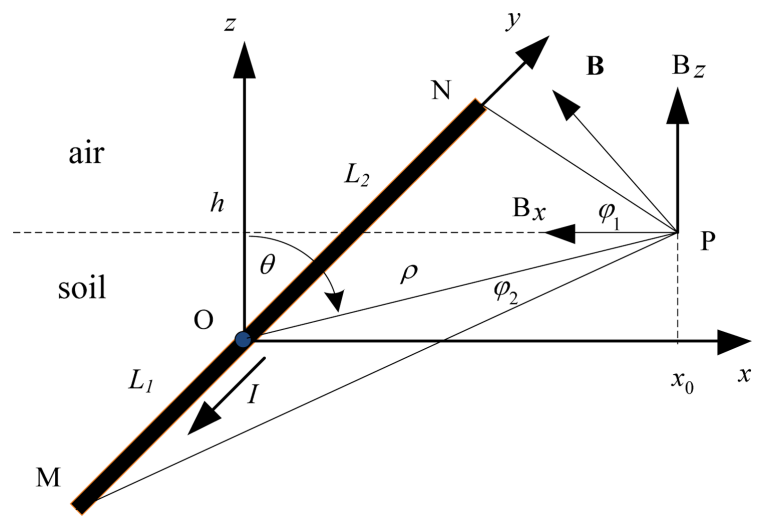

Fig. 1. (Color online) Finite length current-carrying conductor model.
Where, $\sin \varphi_{1}=\frac{L_{2}}{\sqrt{h^{2}+x^{2}+L_{2}^{2}}}, \sin \varphi_{2}=\frac{L_{1}}{\sqrt{h^{2}+x^{2}+L_{1}^{2}}}$

We only take the derivative of $B_{z}(x)$. Eq. (1) includes $\sin \varphi_{1}$ and $\sin \varphi_{2}$, and it is cumbersome to take the derivative of $\sin \varphi_{1}$ and $\sin \varphi_{2}$, so the process of the derivation should be simplified as follows,

When $x \rightarrow 0$,

$$
\lim _{x \rightarrow 0} \sum_{i=1}^{2}\left(d \sin \varphi_{i} / d x\right)=0
$$

The differentiation results of $B_{z}(x)$ are not influenced by $o\left(\sum_{i=1}^{2}\left(d\left(\sin \varphi_{i}\right) / d x\right)\right)$. Similarly, the 1st-, 3rd- and 5thorder derivatives of $B_{z}(x)$ can be expressed by follows equations.

$$
\left\{\begin{array}{c}
B_{z}^{(1)}(x) \approx \frac{\mu I}{4 \pi} \frac{h^{2}-x^{2}}{\left(h^{2}+x^{2}\right)^{2}}\left(\sin \varphi_{1}+\sin \varphi_{2}\right) \\
B_{z}^{(3)}(x) \approx \frac{3 \mu I}{2 \pi} \frac{-h^{4}-x^{4}+6 x^{2} h^{2}}{\left(h^{2}+x^{2}\right)^{4}}\left(\sin \varphi_{1}+\sin \varphi_{2}\right)
\end{array}\right.
$$

Setting $I=1 \mathrm{~A}, h=1 \mathrm{~m}, L 1=L 2=3 \mathrm{~m}$. The $B_{z}(x), 1 \mathrm{st}-$, and 3rd- order derivatives of $B_{z}(x)$ can be calculated and the distribution of the derivatives at $y=0 \mathrm{~m}, y=1 \mathrm{~m}$ and $y=2 \mathrm{~m}$ show in Fig. 2.

It can be seen from Fig. 2 that there is a main peak at the position of the conductor. So if we assume the value of the main peak is $F$, and the value of 1st- and 3rd-order derivatives of $B_{z}(x)$ are $F_{z}^{(1)}$ and $F_{z}^{(3)}$ respectively.

$$
\left\{\begin{array}{c}
F_{z}^{(\mathrm{l})}=\lim _{y \rightarrow 0} B_{z}^{(\mathrm{I})}(y)=I \sum_{i=1}^{2} \frac{\mu L_{i}}{4 \pi h^{2} \sqrt{\left(L_{i}^{2}+h^{2}\right)}} \\
F_{z}^{(3)}=\lim _{y \rightarrow 0} B_{z}^{(3)}(y)=-I \sum_{i=1}^{2} \frac{3 \mu L_{i}\left(2 L_{i}^{2}+3 h^{2}\right)}{4 \pi h^{4}\left(L_{i}^{2}+h^{2}\right) \sqrt{\left(L_{i}^{2}+h^{2}\right)}}
\end{array}\right.
$$

Define

$$
\left\{\begin{array}{c}
\lambda_{z 1}=\sum_{i=1}^{2} \frac{\mu L_{i}}{4 \pi h^{2} \sqrt{\left(L_{i}^{2}+h^{2}\right)}} \\
\lambda_{z 3}=\sum_{i=1}^{2} \frac{3 \mu L_{i}\left(2 L_{i}^{2}+3 h^{2}\right)}{4 \pi h^{4}\left(L_{i}^{2}+h^{2}\right) \sqrt{\left(L_{i}^{2}+h^{2}\right)}}
\end{array}\right.
$$

So that

$$
\left\{\begin{array}{l}
I=\frac{1}{\lambda_{z 1}} F_{z}^{(1)} \\
I=-\frac{1}{\lambda_{z 3}} F_{z}^{(3)}
\end{array}\right.
$$


Table 1. Comparison of the calculation results with the setting values in derivative method.

\begin{tabular}{cccc}
\hline \hline & \multicolumn{2}{c}{ Derivative method } & Real value \\
\cline { 2 - 3 } & $B_{z}{ }^{1}(x)$ & $B_{z}{ }^{3}(x)$ & (A) \\
\hline The value of main peak $F$ & $1.9 \times 10^{-7}$ & $1.2 \times 10^{-6}$ & - \\
Conductor current $I(\mathrm{~A})$ & 1.001 & 1.004 & 1.000 \\
Error (\%) & 0.1 & 0.4 & - \\
\hline
\end{tabular}

When $L_{i}>>h$, Eq. (3) can be simplified as

$$
\left\{\begin{array}{l}
\lambda_{z 1} \approx \sum_{i=1}^{2} \frac{\mu}{4 \pi h^{2}} \\
\lambda_{z 3} \approx \sum_{i=1}^{2} \frac{3 \mu}{2 \pi h^{4}}
\end{array}\right.
$$

Eq. (5) succinctly describe the relationship between conductor current and $F_{z}^{(1)}, F_{z}^{(3)}$, so the conductor current can be directly calculated by Eq. (3) and Eq. (4) when known $F_{z}^{(1)}$ or $F_{z}^{(3)}$. As shown in Table 1, the conductor current can be calculated by the distribution of the derivatives at $y=0$.

Table 1 shows that the setting value of conductor current is $1 \mathrm{~A}$, and the calculation value calculated by $F_{z}^{(1)}$ and $F_{z}^{(3)}$ are $1.001 \mathrm{~A}$ and $1.004 \mathrm{~A}$ with $0.1 \%$ and $0.4 \%$ error respectively. The result indicates that the value of main peaks of derivatives functions of $B_{z}(x)$ can be applied to detect the current flowing through conductor.

\section{Experimental Verification}

\subsection{Lab Experiment}

As showed in Fig. 3, the small model of conductor with Ø3.5 mm copper wire. An F.W. BELL Model 7010 gauss/

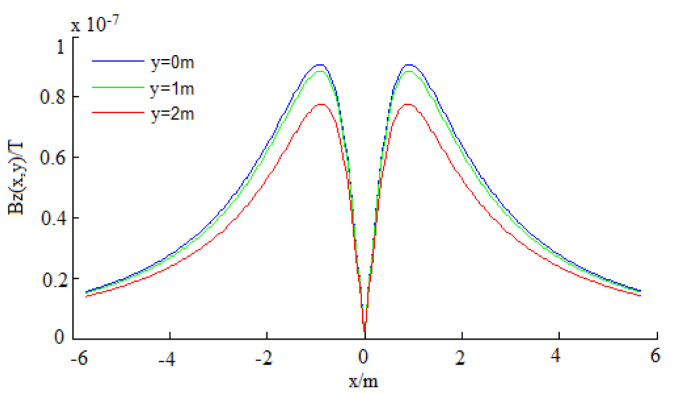

(a) The distribution diagram of $B_{z}(x)$ and section diagram
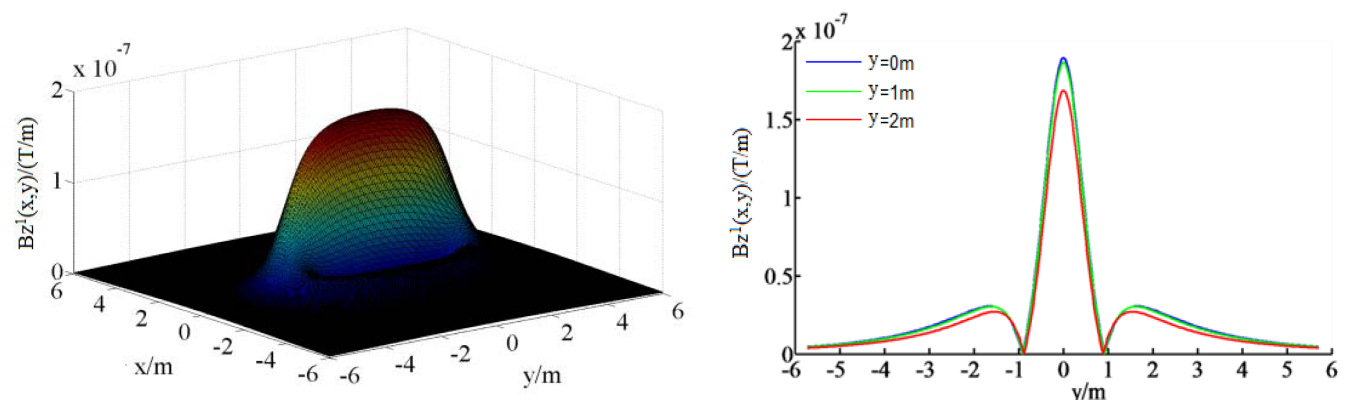

(b) The 1st-order derivatives distribution diagram of $B_{z}(x)$ and section diagram
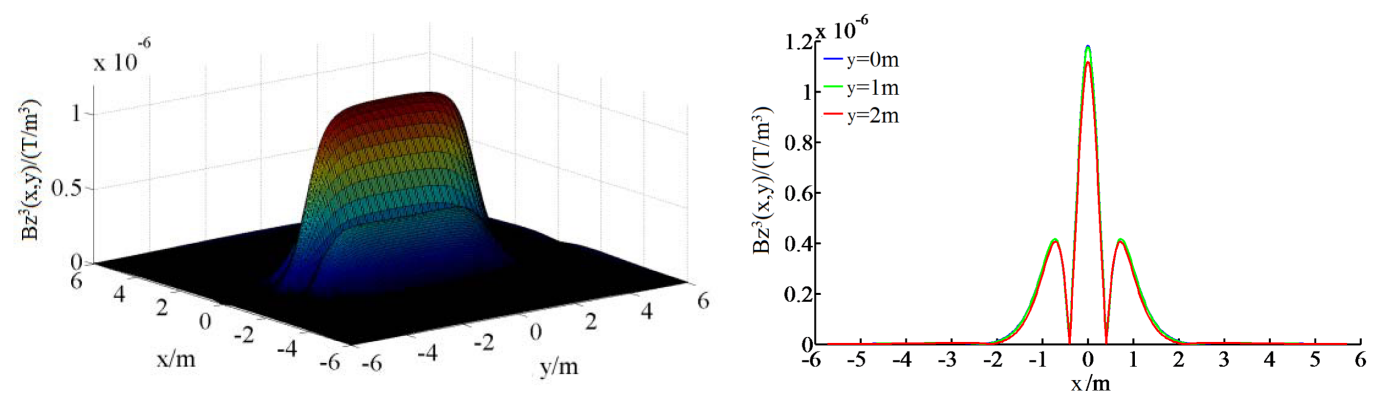

(c) The 3rd-order derivatives distribution diagram of $B_{z}(x)$ and section diagram

Fig. 2. (Color online) The derivative method computing result of $B_{z}(x)$ and section diagram. 


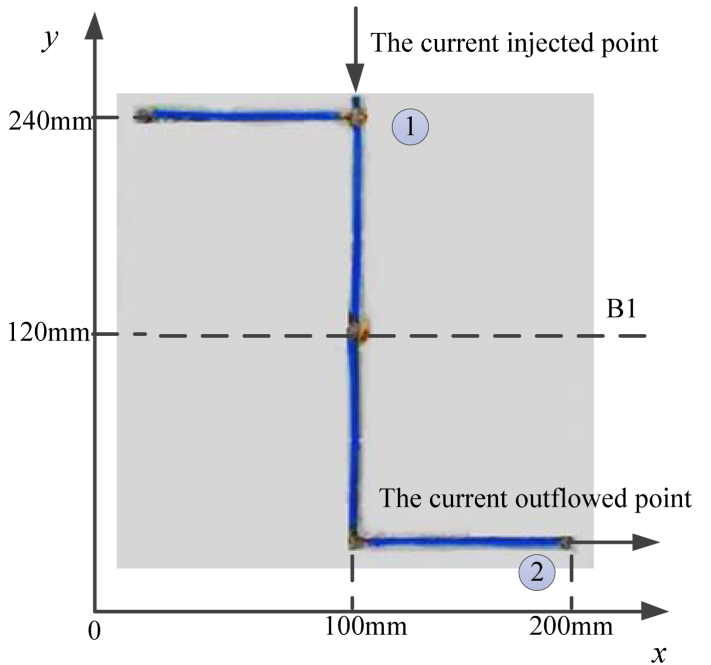

Fig. 3. (Color online) Setup model.

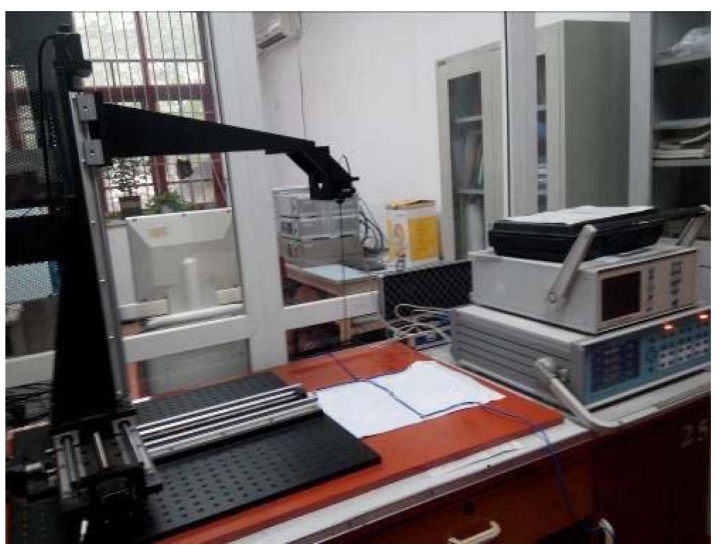

Fig. 4. (Color online) Experiment platform.

tesla meter is used to measure magnetic flux density by utilizing a Hall Effect probe and the probe height from the experimental grid is $h=30 \mathrm{~mm}$. A current of $15.5 \mathrm{~A}$ is injected from node 1 and flowed out from node 2 . A $3 \mathrm{D}$ numerical control motor platform is applied to measure the magnetic flux density in the vertical direction on line $B 1$. For measurement on a line, the total number of measurement points is 200 , the point spacing is $1 \mathrm{~mm}$, the stabilization time of probe at each point is $2 \mathrm{~s}$, and the moving speed of the probe is $0.5 \mathrm{~mm} / \mathrm{s}$ as shown in Fig. 4 .

With the derivative method, the 1st- and 3rd- order derivative of $B_{z}(x)$ is obtained, and a low-pass filter is used to filter noise signal as shown in Fig. 5 and Fig. 6. According to Eq. (3) and Eq. (4), the current in conductor can be calculated as shown in Table 2 .

The Fig. 5 and Fig. 6 show there is a main peaks over the conductors on $B_{z}{ }^{1}(x)$ and $B_{z}{ }^{3}(x)$ respectively, and the current flowing in the conductor can be calculated by the

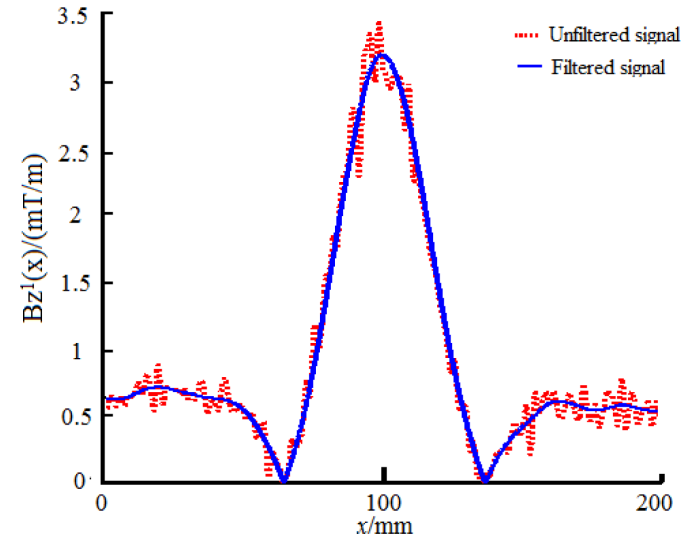

Fig. 5. (Color online) The distribution of $B_{z}(x)^{1}$.

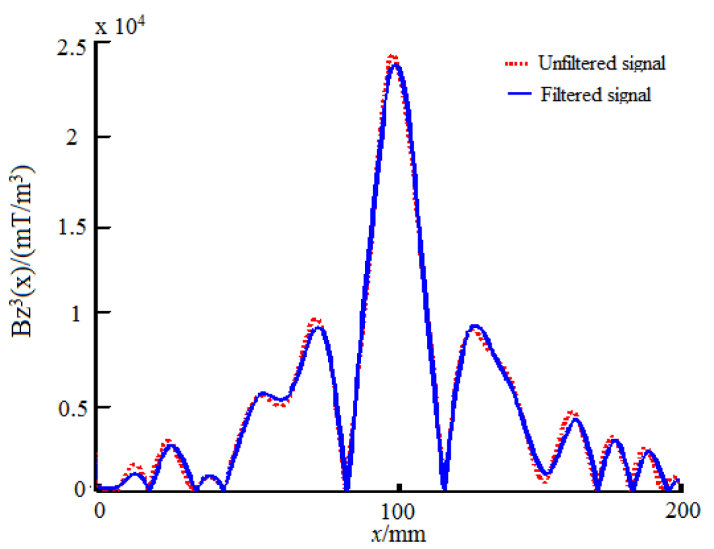

Fig. 6. (Color online) The distribution of $B_{z}(x)^{3}$.

Table 2. Comparison of the calculation results with the setting values in derivative method.

\begin{tabular}{cccc}
\hline \hline & \multicolumn{2}{c}{ Derivative method } & Real value \\
\cline { 2 - 3 } & $B_{z}{ }^{1}(x)$ & $B_{z}{ }^{3}(x)$ & $(\mathrm{A})$ \\
\hline Conductor current $I(\mathrm{~A})$ & 15.9001 & 16.0521 & 15.50 \\
Error $(\%)$ & 2.58 & 3.56 & - \\
\hline
\end{tabular}

method proposed in the paper. According to Eq. (4) and Eq. (5), the main peaks of B1 and B3 is applied to calculate the conductor current. As Table 2 shows that the computing results are $15.9001 \mathrm{~A}$ and $16.0521 \mathrm{~A}$ with 2.58 $\%$ and $3.56 \%$ error, respectively. The results indicate that the derivatives can be used to detect the current flowing through grounding grids with low error.

\subsection{Field experiment}

A field experiment was performed at an abandoned substation as shown in Fig. 7 and Fig. 8. The measuring area is $220 \mathrm{~cm}$ long, there is scale plate marking measuring spots on the measuring area. According to the blueprints of the substation, there is only one conductor of 


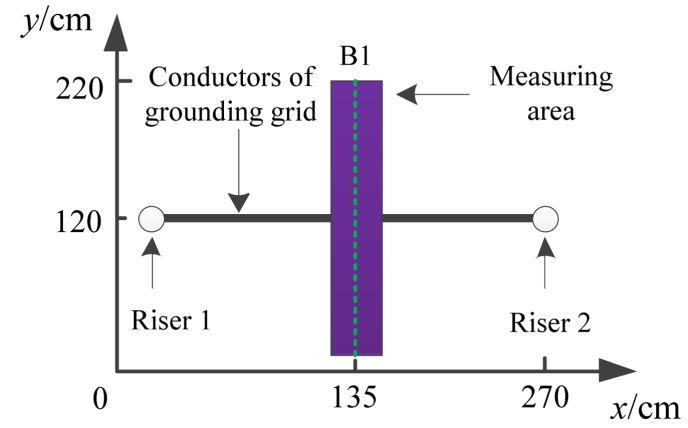

Fig. 7. (Color online) Block diagram of the experimental measurement.

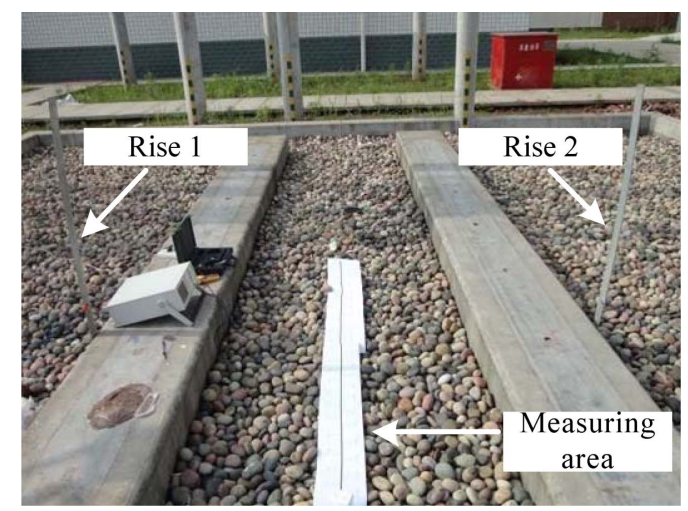

Fig. 8. (Color online) Field experiment.

grounding grid in the measuring area, the conductor connect with two risers, the two risers are away from 270 $\mathrm{cm}$ as shown in Fig. 7, and the buried depth of the grounding grid is $0.8 \mathrm{~m}$.

In the experiment, a current of $19.56 \mathrm{~A}$ is injected from the riser 1 and flowed out from riser 2 as shown Fig. 8. The magnetic flux density in the vertical direction on line B1 is measured. The measurement point spacing is 10 cm. Fig. 9 shows the measuring results.

With the derivative method, the 1st-order derivative of $B_{z}(x)$ is obtained as shown in Fig. 9. Generally, the length

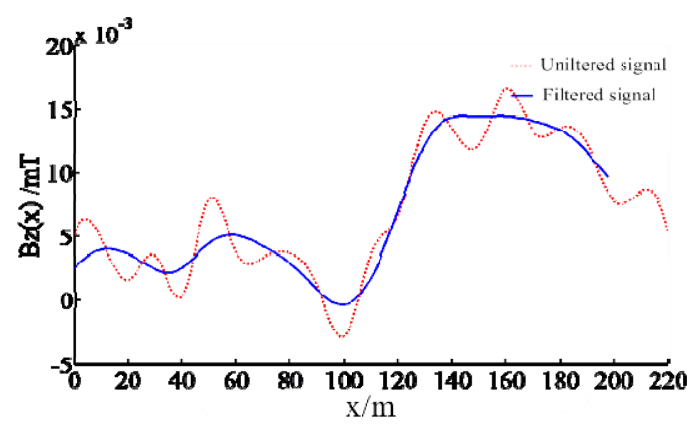

Fig. 9. (Color online) The distribution of $B_{z}(x)$ in measurement region.

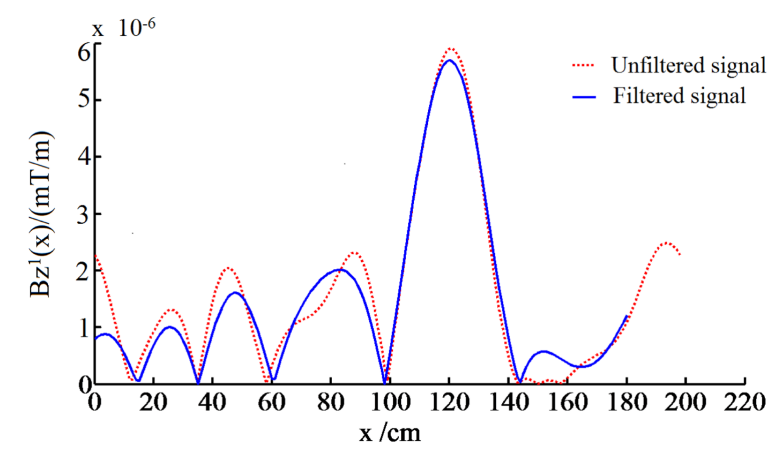

Fig. 10. (Color online) The distribution of $B_{z}(x)^{1}$.

$(L)$ of branch conductor of grounding grid is from $8 \mathrm{~m}$ to $15 \mathrm{~m}$, and the druid depth $(h)$ is form $0.6 \mathrm{~m}$ to $1 \mathrm{~m}$. Due to $L>>h$, the conductor current can be obtained according to Eq. (4) and Eq. (5), and the computing result $I=$ $18.88 \mathrm{~A}$. Comparing with real value $19.56 \mathrm{~A}$, the error of conductor current is $3.48 \%$. The result indicates that the method can detect effectively the current flowing though grounding grids with low error.

\section{Conclusion}

This paper proposed a new method to detect the current flowing through concealed conductor. It is verified that the relationship between the current flowing through conductor and the main peak of the derivative function can be applied to detect the current. The method is applied to detect the conductor current flowing through grounding grids of substation. The lab and field experimental results show that the method can effectively measure the current flowing through grounding grids with low error, which provides a new method to detect current flowing through concealed conductors, such as grounding grids or other.

\section{Acknowledgement}

This work was supported by National Natural Science Foundation of China (51477013) and 2014 Chongqing University Postgraduates' Innovation Project, Project Number: CYS14012; The authors would like to thank the anonymous reviewers for their instructive comments.

\section{References}

[1] X. Zhou and H. Wang, Electric Wire \& Cable. 3, 38 (2005).

[2] M. Li, X. Wang, and H. Pu, J. Beijing Institute of PetroChemical Technol. 4, 49 (2014).

[3] Y. Yang, R. He, and G. Li, Corrosion \& Protection. 4, 
324 (2012).

[4] Z. Bo, Z. Zhibin, and C. Xiang, IEEE Trans. Magn. 38, 473 (2002).

[5] X. Long, M. Dong, and W. Xu, IEEE Trans. Smart Grid. 3, 761 (2012).

[6] R. Alipio, M. A. O. Schroeder, and M. M. Afonso, IEEE Trans. Ind. Appl. 51, 4912 (2015).

[7] F. E. Asimakopoulou, G. J. Tsekouras, and I. F. Gonos, Electron. Power Syst. Res. 94, 113 (2013).

[8] V. R. Lawson, IEEE Trans. Ind. Appl. 24, 25 (1988).
[9] P. H. Zhang, J. J. He, and D. D. Zhang, Metrology \& Measurement Systems. 19, 63 (2012).

[10] Y. Ma, G. G. Karady, and S. Kucuksari, IEEE Power \& Energy Society General Meeting (2010) pp 1-6.

[11] F. Dawalibi, IEEE Trans. Power Deliver. 1, 112 (1986).

[12] Y. Liu, X. Cui, and Z. Zhao, Frontiers of Electrical \& Electronic Engineering in China. 5, 501 (2010).

[13] B. Zhang, Z. Zhao, and X. Cui, IEEE Trans. Magn. 38, 473 (2002). 